\title{
A Frequency Domain Transformer Model for Simulating Fast Transient Overvoltages
}

\author{
V. VENEGAS, J.L. GUARDADO, E. MELGOZA, A. GONZALEZ \\ Department of Electrical Engineering \\ Instituto Tecnológico de Morelia \\ Av. Tecnológico 1500, Col. Lomas de Santiaguito, C.P. 58120 \\ MORELIA, MEXICO
}

\begin{abstract}
In this work a computer model for calculating surge distribution in transformer windings is presented. The transformer model is developed using multiconductor transmission line theory. The capacitance, inductance, resistance and conductance are calculated with simple formulations and these parameters are used for calculating modal parameters in order to represent the winding as a two port network. The model is validated by means of a comparison between measured and calculated voltages in a power transformer disk with 16 turns.
\end{abstract}

Key-Words:- Electrical parameters, modal parameters, fast transients, transformer windings.

\section{Introduction}

The transformer is one of the most important and expensive equipment in power systems. This has originated several studies for understanding its behavior, specially during switching transients or lightning, which can cause different kinds of failures [1,2,3]. The Mexican Utility (CFE) reports that $53 \%$ of the failures in power transformers occur in the insulation system and the major percent is due to the presence of electromagnetic transients [3].

The transient overvoltages produce high dielectric stresses that can damage the turn insulation, which can lead to major failures like short circuit between turns, layers or disks of the transformer [3]. The surges have two characteristics that can be dangerous for the insulation winding: short rate of rise and high amplitude. Typical rates of rise are in the order of 10 to $20 \mathrm{~ns}$ and the amplitudes are in the order of 1.5 to 2.5 p.u. [2]. Short rates of rise are responsible of a nonlinear voltage distribution in the winding, which may lead to failures on winding sections nearest to transformer terminals.

Several computer models have been proposed for studying electromagnetic transients in transformers. The most of models are based on equivalent lumped circuits with coupled inductances and capacitances $[4,5]$. Some models have been developed using transmission line theory, which has the advantage of including more easily the frequency dependent phenomena and the distributed parameters of the winding [2,6].
The aim of this work is to develop a transformer model in the frequency domain for calculating surge distribution in power transformer windings. The model is developed by means of the transmission line theory and modal analysis and takes into account the frequency dependent resistance of the cooper conductors. The results of the voltage transients calculated at the turns of a winding disk were compared with laboratory measurements.

\section{Model Description}

The frequency domain transformer winding model is developed using the multiconductor transmission line theory. The propagation of voltages and currents along the transmission line is given by:

$$
\begin{aligned}
& \begin{array}{l}
d^{2} \boldsymbol{V} \\
d x^{2}
\end{array}=\boldsymbol{Z} \boldsymbol{Y} \boldsymbol{V}=\boldsymbol{P} \boldsymbol{V} \\
& d^{2} \boldsymbol{I} x^{2}=\boldsymbol{Y} \boldsymbol{Z} \boldsymbol{I}=\boldsymbol{P}_{\boldsymbol{T}} \boldsymbol{I}
\end{aligned}
$$

where:

$\boldsymbol{V}, \boldsymbol{I}$.- Voltage and current vectors.

$Z$.- $\quad$ Series impedance matrix.

$\boldsymbol{Y}$.- $\quad$ Parallel admitance matrix.

$\boldsymbol{P}$.- $\quad$ Product of matrices $\boldsymbol{Z}$ and $\boldsymbol{Y}$.

$\boldsymbol{P}_{\boldsymbol{T}}$.- $\quad$ Product of matrices $\boldsymbol{Y}$ and $\boldsymbol{Z}$. 
For developing the model a single turn is considered as the basic element of analysis. Fig. 1 shows a transformer winding with three turns in series connection. In the figure, $Z_{r}$ is the impedance for representing the terminal conditions: short and open circuit. The voltage $V_{s 1}$ is the surge at the beginning of the winding.



Fig. 1. Winding model with three turns in series connection.

From modal analysis, the terminal conditions of the model are given by:

$$
\left[\begin{array}{c}
I_{s 1} \\
I_{s 2} \\
I_{s 3} \\
I_{r 1} \\
I_{r 2} \\
I_{r 3}
\end{array}\right]=\left[\begin{array}{rr}
\boldsymbol{A} & -\boldsymbol{B} \\
-\boldsymbol{C} & \boldsymbol{D}
\end{array}\right]\left[\begin{array}{l}
V_{s 1} \\
V_{s 2} \\
V_{s 3} \\
V_{r 1} \\
V_{r 2} \\
V_{r 3}
\end{array}\right]
$$

where $\boldsymbol{A}, \boldsymbol{B}, \boldsymbol{C}$ y $\boldsymbol{D}$ are modal matrices of the winding and are given by:

$$
\begin{aligned}
& \boldsymbol{A}=\boldsymbol{D}=\boldsymbol{Y}_{\mathbf{0}} \operatorname{coth}(\sqrt{\mathbf{Z} \boldsymbol{Y} l)} \\
& \boldsymbol{B}=\boldsymbol{C}=\boldsymbol{Y}_{\boldsymbol{0}} \operatorname{cosch}(\sqrt{\mathbf{Z Y}} l)
\end{aligned}
$$

The following voltage and current relations can be written from Fig. 1:

$$
\begin{array}{cc}
I_{r 1}=-I_{s 2} & I_{r 2}=-I_{s 3} \quad V_{r 1}=V_{s 2} \\
V_{r 2}=V_{s 3} & -I_{r 3}=V_{r 3} / Z_{r}
\end{array}
$$

Substituting equations (6) in (3) and reducing through matrix operations, the terminal voltages and currents are given by [7]:

$$
\left[\begin{array}{l}
I_{s 1} \\
V_{s 2} \\
V_{s 3} \\
V_{r 3}
\end{array}\right]=\left[\begin{array}{ll}
\boldsymbol{M} \\
\end{array}\right]\left[\begin{array}{l}
V_{s 1} \\
0 \\
0 \\
0
\end{array}\right]
$$

Equation (7) is used for calculating surge distribution multiplying $V_{s 1}$ and the first column of the system matrix $\mathrm{M}$. The transient overvoltages are transformed to the time domain using the FFT algorithm.

\section{Electrical Parameters Calculation}

For calculating the modal parameters is necessary to have the matrices $\boldsymbol{Z}$ and $\boldsymbol{Y}$, which are calculated by the general formulations:

$$
\begin{aligned}
& Z=R+j \omega \boldsymbol{L} \\
& \boldsymbol{Y}=\boldsymbol{G}+\mathrm{j} \omega \boldsymbol{C}
\end{aligned}
$$

where $\boldsymbol{R}, \boldsymbol{L}, \boldsymbol{G}$ y $\boldsymbol{C}$ are de resistance, inductance, conductance and capacitance matrices, respectively.

The capacitance is determined using the parallel plate approach, then [4]:

$$
C=\frac{\varepsilon_{0} \varepsilon_{r} A}{d}
$$

where $\varepsilon_{r}$ is the relative permitivity of the insulation, $A$ is the area of the winding section involved and $d$ is the distance between the surfaces. The capacitance matrix is form using the capacitances to ground and the coupling capacitances between adjacent turns. Fig. 2 shows a transformer disk with $n$ turns and some of the capacitances considered.

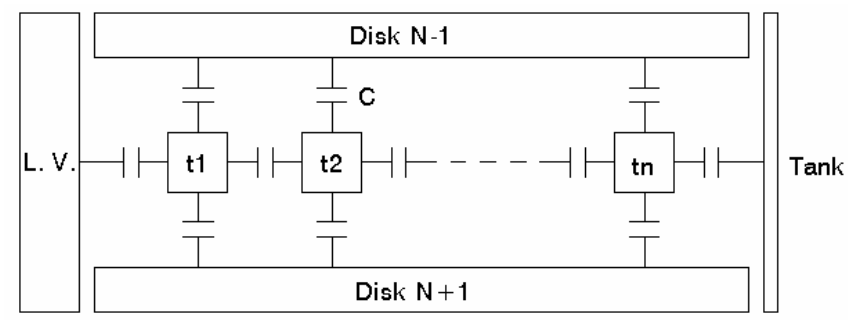

Fig. 2. Coil under study with $n$ turns. 
Once the capacitance is calculated, the conductance matrix is obtained with:

$$
G=\omega C \tan \delta
$$

where $\tan \delta$ is the loss tangent of the turn insulation.

The inductance is calculated considering that the turns are similar to a lossless transmission line where the propagation depends on the dielectric and magnetic properties of the winding, then [6]:

$$
\mathbf{L}=\frac{1}{v_{s}^{2}} C^{-1}
$$

where $v_{s}$ is the velocity of the wave propagation given by:

$$
v_{s}=\frac{1}{\sqrt{\mu \varepsilon}}
$$

The frequency dependent resistance for each turn is calculated with [8]:

$$
R_{\text {skin }}=\frac{l}{a \delta \sigma}
$$

where $\sigma$ is the conductivity, $l$ the turn lenght and $a$ the perimeter of the transversal section of one turn. The term $\delta$ is the magnetic flux penetration into conductor regions due to skin effect:

$$
\delta=\sqrt{\frac{2}{\omega \sigma \mu}}
$$

\section{Results}

In order to validate the computer model, a comparison between the transient voltages calculated and measured in a power transformer coil with 16 turns was carried out. A recurrent surge generator was used to apply steep fronted waves of approximately $10 \mathrm{~ns}$ and $22 \mathrm{~V}$ crest. The turn to ground voltages were measured at each turn with the remote end of the disk left in short circuit and open circuit conditions.
Figs. 3 and 4 show simulations and measurements for different turns for a period of time of $400 \mathrm{~ns}$. Voltages for short circuit conditions are presented in Fig. 3, while in Fig. 4 the voltages for open circuit conditions are shown.

From figures 3 and 4 it is clear that the computer model is capable of calculating the transient voltages with a reasonable degree of accuracy. In general, there exist good correlation on the shape and magnitude of the surge distribution calculated and measured. This gives confidence in the assumptions followed for developing the distributed parameter model.

The results show that the surge propagation inside the winding is similar to that of the travel waves in transmission lines. The applied pulse penetrates into the coil where it is attenuated, distorted and reflected until it arrives to the remote end. For short circuit conditions the magnitude of the pulse is reduced while it travels from turn to turn. The peak magnitude is increased gradually while the voltage travels inside the coil until it reaches the final end. The major magnitude occurs at this point due to the great difference between the characteristic impedance of the disk and the final impedance $(\mathrm{Zr}=\infty)$.

\section{Conclusions}

In this work a trasformer model for calculating surge propagation is presented. The model is based on the transmission line theory. The electrical parameters are determined with simple formulations that takes into account the electric and magnetic characteristics of the winding.

The computer model was validated by a comparison between measured and calculated voltages in a power transformer disk with 16 turns. The results show that the model is adequate for calculating voltage propagation for short periods of time $(<1.2 \mu \mathrm{s})$.

The technique described is useful for developing transformer models valid for longer periods of time, which most take into account magnetic flux penetration into the iron core and electromagnetic coupling between disks. Future works should address this point.

\section{Acknowledgements}

The present work was supported by ANUIES and DGEST of México. 


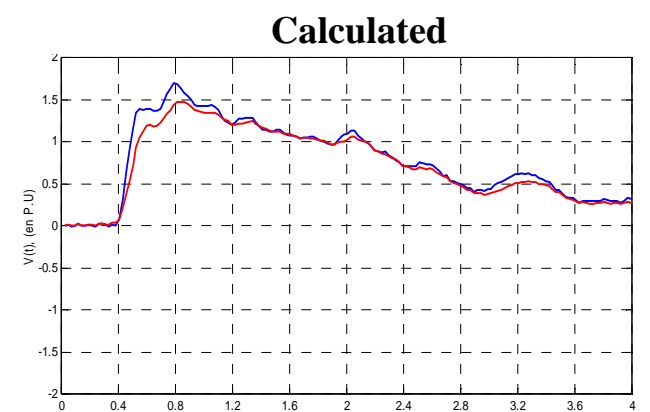

Applied (Blue) - Turn 1(Red)

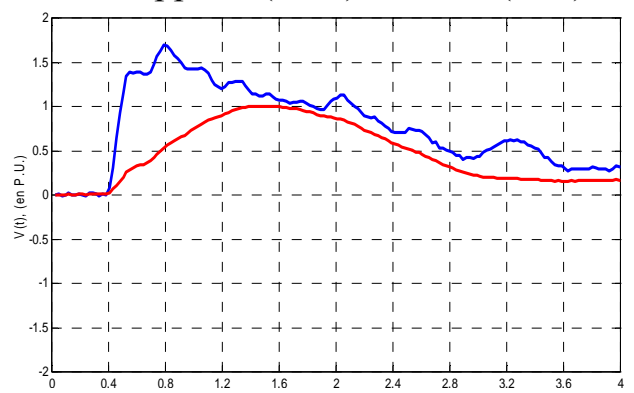

Applied (Blue) - Turn 6(Red)



Applied (Blue) - Turn 9(Red)

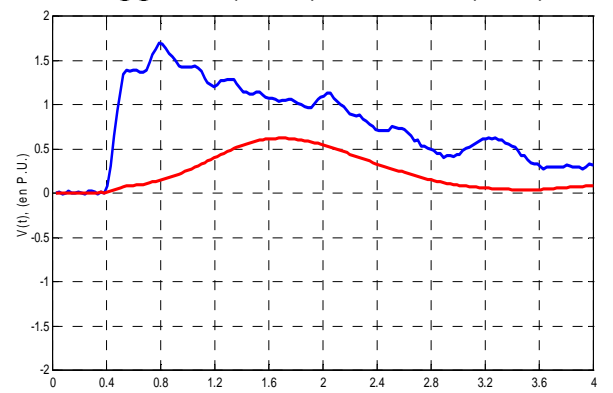

Applied (Blue) - Turn 12(Red)

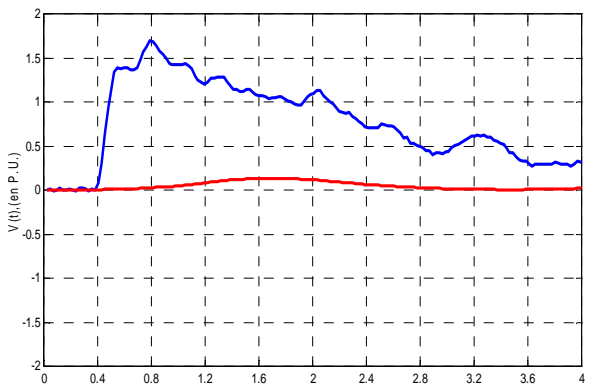

Applied (Blue) - Turn 15(Red)
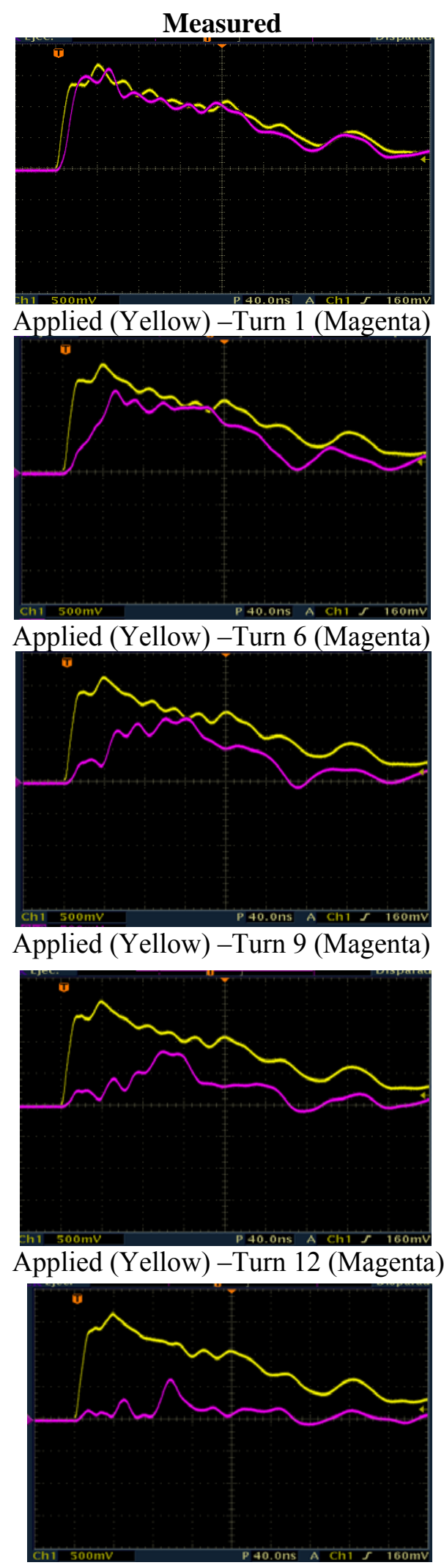

Applied (Yellow) -Turn 15 (Magenta)

Fig. 3 Surge distribution calculated and measured. Short circuit conditions. 


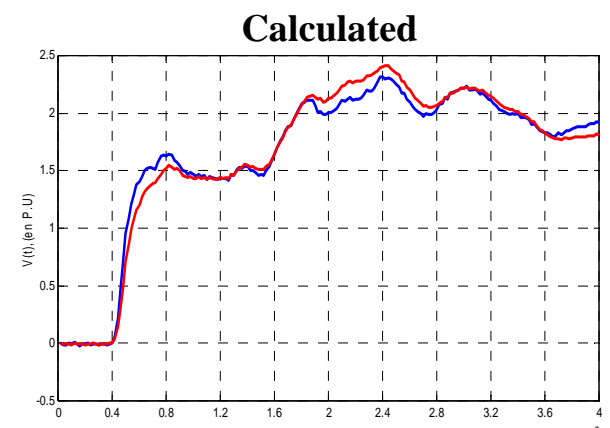

Applied (Blue) - Turn 1 (Red)

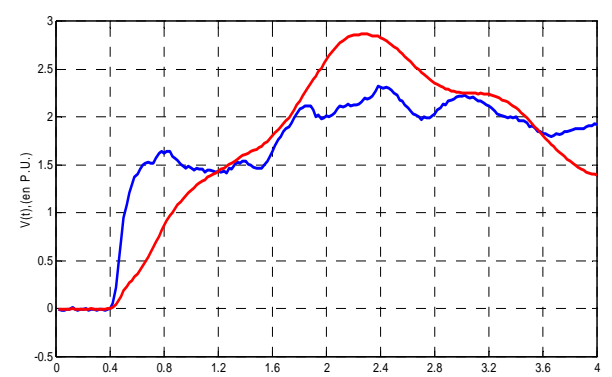

Applied (Blue) - Turn 6 (Red)



Applied (Blue) - Turn 9 (Red)
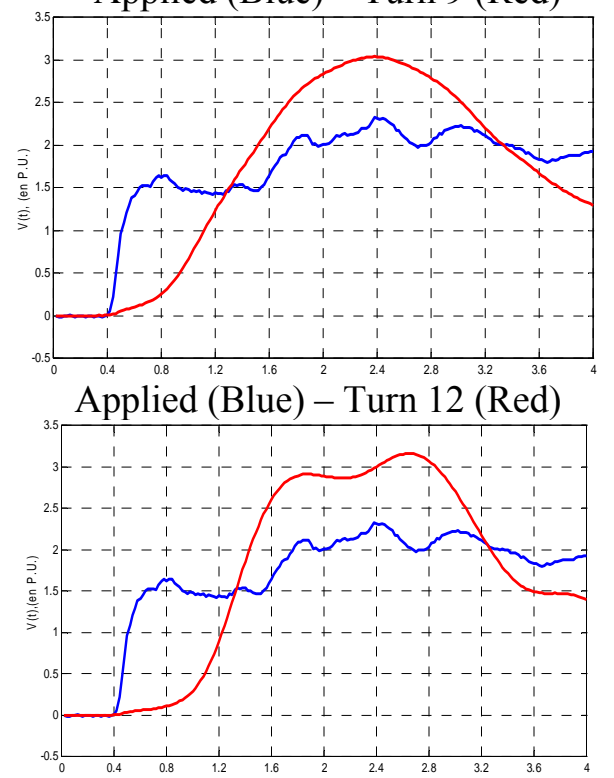

Applied (Blue) - Turn 16 (Red)
Measured
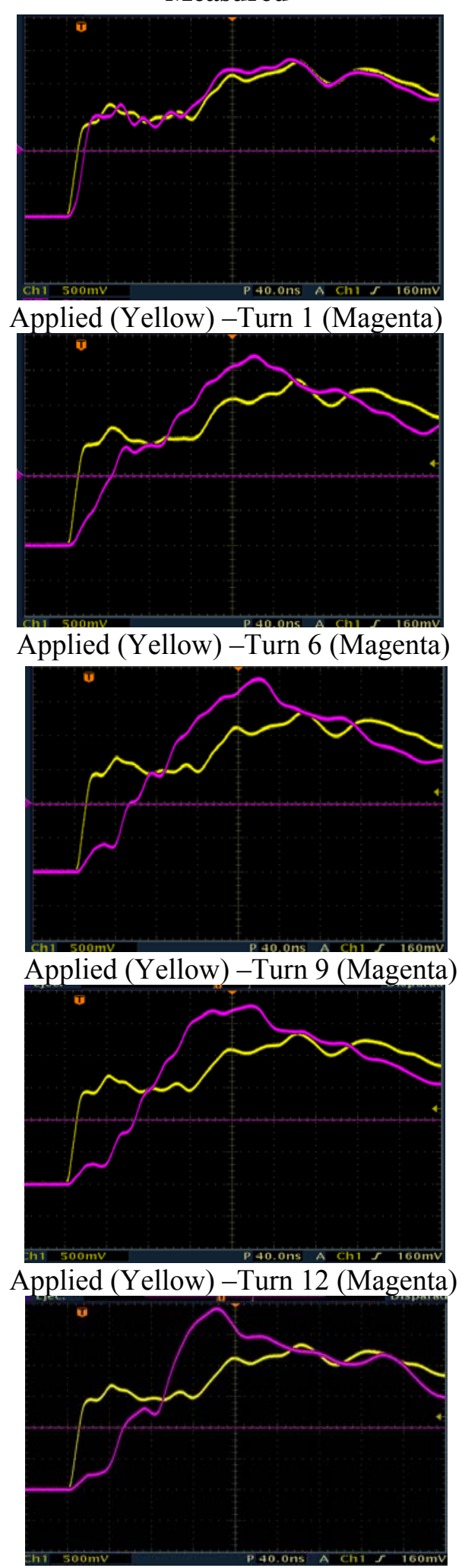

Applied (Yellow) -Turn 16 (Magenta)

Fig. 4 Surge distribution calculated and measured. Open circuit conditions. 
References:

[1] K. J. Cornick, A.M. Kunji, "Nanosecond switching transients recorded in a mining transformer installation", Paper 92 SM 576-9 PWRD, IEEE 1992.

[2] K. J. Cornick, B. Filliat, C. Kieny, W Muller, "Distribution on very fast transient overvoltages in transformer windings", presented at CIGRE, paper 12-116, Paris, 1992.

[3] Mexican Utility (CFE), "Failures in power transformers", technical report, March 1999.

[4] Y. Shibuya, S. Fujita, "High frequency model and transient response of transformer windings", paper 0-7803-7525-4, IEEE 2002, pp. 1839-1844.

[5] E. Bjerkan, H.K. Hoidalen, "High frequency FEMbased power transformer modeling: Investigation of internal stresses due to network-initiated overvoltages", presented at the International Power System Transients IPST, paper IPST05-106, Montreal, Canada, June 2005.

[6] M. Popov, L. van der Sluis, G. Paap, H. de Herdt, "Computation of very fast transient overvoltages in transformer windings", presented at the International Power System Transients IPST, paper IPST05-009, Montreal, Canada, June 2005.

[7] J.L. Guardado, V. Venegas, E. Melgoza, K.J. Cornick and J.L. Naredo, "Transient overvoltages in electrical motors during sequential pole closure," IEEE Trans. Energy Conversion, Vol. 14, No. 4, December 1999.

[8]. J.L.Guardado, K.J. Cornick, "Calculation of machine winding electrical parameters at high frequencies for switching transient studies", IEEE Trans. on Energy Conversion, Vol 2, No. 1, 1996, pp. 33-40. 